\title{
Pharmacophore Mapping and Virtual Screening for SIRT1 Activators
}

\author{
Sugunadevi Sakkjah, Navaneethalsishnan Krishnamoorthy, Poomima Gajendrarao, Sundarapandian Thangapandian, \\ Yuno Lee, Songmi Kim, Jung-Keun Suh, ${ }^{\dagger}$ Hỵong-Ha Kim, ${ }^{\ddagger-}$ and Keun Woo Lee
}

\author{
Division of Applied Life Science (BK21 Program). Environmental Biotechnologv National Core Research Center(EB-NCRC), \\ Plant Holectilar Biology and Biotechnologv Research Center (PMBBRC), Gyeongsang National Lniversity, \\ Jinit 660-701, Korea. "E-mail: kwlee agmuack \\ ${ }^{\dagger}$ Bio Computing Major, Korean German Institute of Technology, Seoul 157-033, Korea \\ ${ }^{\ddagger}$ Division of Ouality of Life, Center of Bioanalysis, Korea ReSearch Institute of Standards and Science (KRISS), \\ Daejeon 305-340, Korea \\ Received December 20, 2008, Accepted April 3, 2009
}

\begin{abstract}
Silent information regulator $2(\operatorname{Sir} 2)$ or sirtuins are $\mathrm{NAD}(+)$-dependent deacetylases, which hydrolyze the acetyllysine residues. In mamnals, sirtuins are classified into seven different classes (SIRT l-7). SIRT l was reported to be involved in age related disorders like obesity, metabolic syndrome, type II diabetes mellitus and Parkinson's disease. Activation of SIRT 1 is one of the promising approaches to treat these age related diseases. In this study, we have used HipHop module of CATALYST to identify a series of phamacophore models to screen SIRT l enhancing molecules. Three molecules from Sirtris Pharmaceuticals were selected as training set and 607 sirtuin activator molecules were used as test set. Five different hypotheses were developed and then validated using the training set and the test set. The results showed that the best pharmacophore model has four features, ring aromatic, positive ionization and wo hydrogen-bond acceptors. The best hypothesis from our study, Hypo2, screened high number of active molecules from the test set. Thus, we suggest that this four feature phamacophore model could be helpful to screen novel SIRTI activator molecules. Hypo2-virtual screening against Maybridge database reveals seven molecules, which contains all the critical features. Moreover, two new scaffolds were identified from this study These scaffolds may be a potent lead for the SIRT I activation.
\end{abstract}

Key Words: Sirtuin Sirtuin Activator. Pharmacophore. HipHop. SIRT 1

\section{Intoduction}

Sirtuin belongs to the class III listone deacetylase (HDAC) family. This HDACs are classified into four different classes: class I contains HDACs 1-3 and 8, class II contains HDACs 4-7. 9 and 10. class III contains sirtuins and class IV contains HDAC 11. Several reports support that sirtuins are $\mathrm{NAD(+)-}$ dependent histone deacety lase proteins. ${ }^{-\cdot 4}$ To date, in human seven types of sirtuins have been identified and they all shares the same catalytic domain but differ in $\mathrm{N}$ - and C-terminals. SIRT 1. 2. 3. 5 and 6 are NAD dependent deacetylases. SIRT4 and 6 are ADP-ribosyltransferases. ${ }^{5.6}$ In addition, SIRT7 is involved in both ADP-ribosyltransferase and rDNA transcription. Sirtuins play a major role in deacetylation that couples lysine deacetylation to NAD+ hydrolysis to produce the deacetylated substrate O-acetyl-ADP-ribose and nicotinamide. ${ }^{3}$

SIRT I plays a vital role in insulin signaling and glucose metabolism thus it is an excellent target to treat type II diabetes. obesity and metabolic syndrome by enhancing its function. ${ }^{8.9}$ Except SIRT 1 all other sirtuins (SIRT2-7) consist of approximately 400 amino acids. whereas SIRT1 consists of 744 amino acids. It composed of two domains. namely, Rossman fold (large) domain and zinc binding (small) domain. NAD is a co-factor for sirtuins where it binds to the cleft between the two domains (large and small domain). immediately adjacent to the acetylated peptide binding tumnel. ${ }^{30}$ The N-terminal amino acids G183-I225 play a critical role to enhance the activity. $^{11,12}$

Sirtuins are vastly expressed in several adult tissues such as brain, heart and skeletal nuscle. SIRT1 is known to interact with several inportant transcription factors like p53, p65 (NF-kB). MýoD and regulates their activities. ${ }^{13}$ Therefore. sirtuins are crucial targets to design activators and inhibitors. There are two potential conceptual modes for activating sirtuins: direct and allosteric methods. ${ }^{14}$ In this work, we mainly focus on allosteric method to design activator molecules for SIRT1. Some activators have been reported. e.g.. resveratrol, fisetin and butein, but all these compounds are plant polyphenols. ${ }^{1516}$ Resveratrol is a natural substrate. which can be found in red wine and activates SIRT1 and extends lifespan of mammals. ${ }^{1 ₹ .18}$

The aim of this study is to generate a pharmacophore model based on conmon features of molecules, which could enlance the SIRTI activity. It was achieved using HipHop module from the CATALYST software. ${ }^{19}$ The constructed pharnacophore model has been used for screening the large database to identify the new molecules that are presumably able to activate SIRTI. We found that our four feature pharmacophore model (Hypo2) screened large number of highly active molecules of SIRT1 activators from the test set. Among these four features in Hypo2. the ring aromatic and the positive ionization features appear to play a vital role for the molecules to be a SIRTI activator. Virtual screening revealed seven molecules (two scaffolds) with all the required critical features. 


\section{Methods}

Pharmacophore Modeling. Pharmacophore modeling is one of the most powerful techniques to classify and identify key features from a group of molecules such as active and inactive compounds. Chemical features in the hypothesis or pharmacophore model will furnish a new insight to design novel molecules that can enhance or inhibit the function of the target and will be useful in drug discovery strategies. HipHop module from CATALYST software was used to develop phammacophore models. To date. there is no three-dimensional structural information for SIRTI. especially for the allosteric site. Therefore the common feature pharmacophore modeling method was adopted to predict the potency of SIRT1 activators.

In order to generate a pharmacophore model three molecules were taken from Sirtris Pharmaceuticals as a training set (Fig. 1) ${ }^{2(j)}$ All compounds were built using ISIS draw and minimized using CATALYST software to the closest local minimum by applying the Charmm-like force field. ${ }^{21}$ Maximum number of 250 conformers were generated utilizing the best conformational analysis with an energy threshold of 20

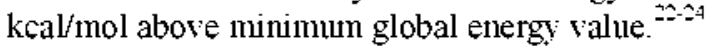

Different types of chemical features for each molecule in the training set were examined using feature dictionary from CATALYST software. By consolidating all the available features. six common features were used to generate the hypothesis: hydrogen-bond acceptor ( $H B A$ ), hydrogen-bond donor $(H B D)$, hydrophobe $(H)$. hydrophobe aromatic $(Z)$. ring aromatic $(R A)$ and positive ionization $(P)$. The training set was taken for HipHop module to generate the common feature hypothesis. Five different hypotheses were generated (Table 1) with different combination of the above features. These five hypotheses were validated using the test set which includes 607 molecules having a wide range of activity values. Based on the activity values the test set molecules were divided into four different sets. The hypothesis that screened high number of molecules from the active set and low number of molecules from the inactive set are considered to be the best hypothesis to screen SIRT I activators.

Database Search for New Hits. In order to find novel molecules. which include all the desired chemical features to activate SIRT1, the best hypothesis model. Hypo2 was used to screen Maybridge database available in CATALYST software. The Best Flexible Search Database/Spread Sheets method in CATALYST was used for the database search to find out similarly featured molecules. The hit molecules from Maybridge were further refined by calculating the solubility. absorption and Lipinski's rule-of-five to avoid false positives. In order to validate the good quality of our hypothesis. we screened the compounds that are related to our chemical features and the resultant molecules were superimposed with the training set molecules. ${ }^{26}$

\section{Result and Discussion}

Training Set and Test Set Constluction. Three molecules were taken from Sirtris Phamnaceuticals as a training set to generate a series of pharmacophore models (Fig. l). In order to evaluate and validate the generated pharnacophore models a test set was prepared and cross validated. ${ }^{25}$ The 607 molecules. which are structurally different from the training set were selected to prepare the test set. We sorted the test set molecules into four different sets depending on the activity data. They are active (A). moderate (M), least active (LA) and inactive (IA) set. Active set contains 176 most active com pounds within the range of $0-5 \mathrm{HM}$. the moderate set contains 191 moderately active compounds within the range of 5-50 $\mu \mathrm{M}$, the least active set contains 58 least active molecules within the range of $150-200 \mu \mathrm{M}$ and the rest 182 inactive molecules are classified as an inactive set.

Evaluation of Hypotheses. In this study, we have generated

Table 1. Chemical feature compositions for the five hypotheses

\begin{tabular}{cccccccc}
\hline Name & $\begin{array}{c}\text { No. of } \\
\text { Features }\end{array}$ & $H B .1$ & $H B D$ & $R-1$ & $Z$ & $H$ & $P$ \\
\hline Hypol & 3 & 2 & 1 & 0 & 0 & 0 & 0 \\
Hypo2 & 4 & 2 & 0 & 1 & 0 & 0 & 1 \\
Hypo3 & 5 & 2 & 1 & 0 & 0 & 1 & 1 \\
Hypot & 6 & 2 & 1 & 0 & 1 & 2 & 0 \\
Hypo5 & 7 & 3 & 0 & 0 & 1 & 2 & 1 \\
\hline
\end{tabular}

HBA: Hydrogen Bond Acceptor, HBD: Hฯdrogen Bond Donor, RA: Ring Aromatic. Z: Hydrophobe Aromatic. H: Hydrophobe, P. Positive Ionization.<smiles>Oc1ccc(/C=C/c2cc(O)cc(O)c2)cc1</smiles>

Resveratnol $\left(\mathrm{EC}_{1} \leqslant 46 \mu \mathrm{M}\right)$

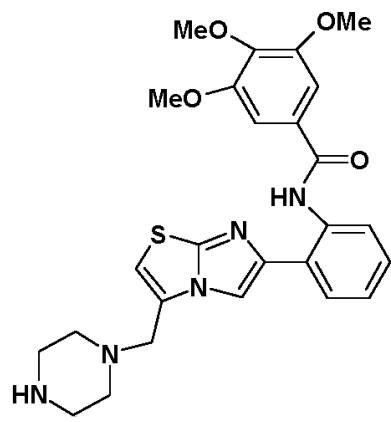

SRT $1460\left(\mathrm{EC}_{1} \leq 2.90 \mu \mathrm{M}\right)$

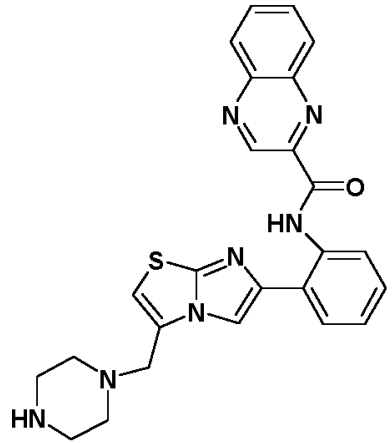

$\operatorname{SRT1720}\left(\mathrm{EC}_{1.5} 0.16 \mu \mathrm{M}\right)$

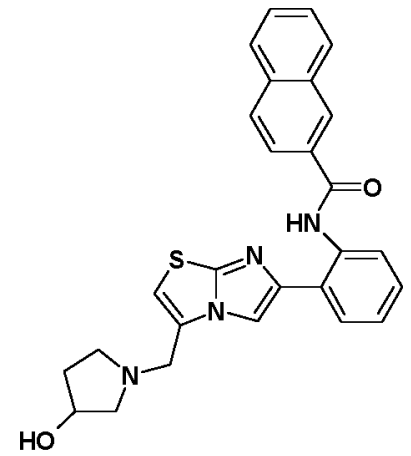

SRT2183 $\left(\mathrm{EC}_{1.5} 0.36 \mu \mathrm{M}\right)$

Figure 1. Standard molecules for training set. Chemical structures of three molecules from Sirtris Pharmaceutical which are used as training set (SRT1720, SRT 1460 and SRT2183). Reseveratrol is a natural substrate for sirtuinl activator. ${ }^{2(1}$ 
Table 2. The numbers of molecules screened by each hypothesis

\begin{tabular}{ccccc}
\hline Phannacophore & $\begin{array}{c}\text { A set } \\
(176)\end{array}$ & $\begin{array}{c}\text { M set } \\
(191)\end{array}$ & $\begin{array}{c}\text { LA set } \\
(58)\end{array}$ & $\begin{array}{c}\text { LA set } \\
(182)\end{array}$ \\
\hline Hypol & 20 & 17 & 6 & 7 \\
Hypo2 & 47 & 45 & 5 & 10 \\
Hypo3 & 5 & 1 & 0 & 0 \\
Hypo4 & 1 & 2 & 1 & 1 \\
Hypo5 & 1 & 0 & 0 & 0 \\
\hline
\end{tabular}

A Set: Active Set. M set: Moderated set LA set: Least Active set. IA set: InActive set.

five different hypotheses (Table 1) based on the training set. All the five hypotheses were generated using HipHop module from CATALYST software. The test set is used to examine the pharmacophore model. The test set contains 607 structurally distinct molecules from the training set, which were prepared adopting the same protocol as the training set molecules were prepared. The best lypothesis was selected depending on the number of the molecules. which were screened from the four different sets constructed based on the activity values of the test set molecules (Table 2).

The Hypol screened 20 molecules out of 176 molecules from A set. 17 molecules out of 191 molecules from M set. 6 molecules out of 58 molecules from LA set and 7 molecules out of 182 molecules from IA set. The highest numbers of molecules were screened from $\mathrm{A}$ and $\mathrm{M}$ set (Fig. 2). To identify the essential features and to increase the quality of the phammacophore. we refined this hypothesis by adding and deleting some of the features like RA, P. $Z$ and $H B D$. In the first modification. $H B D$ group was obliterated and two features. one $R A$ and one $P$ are added in Hypo2 (Fig. 3). It screened +7 molecules out of 176 molecules from A set. 45 molecules out of 191 molecules from $\mathrm{M}$ set. 5 molecules out of 58 molecules from LA set and 10 molecules out of 182 molecules from IA set. After the modification of these two features. $R A$ and $P$. Hypo 2 screened high quantity of molecules from A set. Comparison of Hypol and Hypo 2 revealed that hypo 2 screened more number of molecules from $A$ and $M$ set and at the same time it screened less number of molecules from LA and IA set. Based on this comparison result, we speculate that $R A$ and $P$ features are essential for SIRT1 activity. In order to find which group ( $R A$ or $P$ ) play a crucial
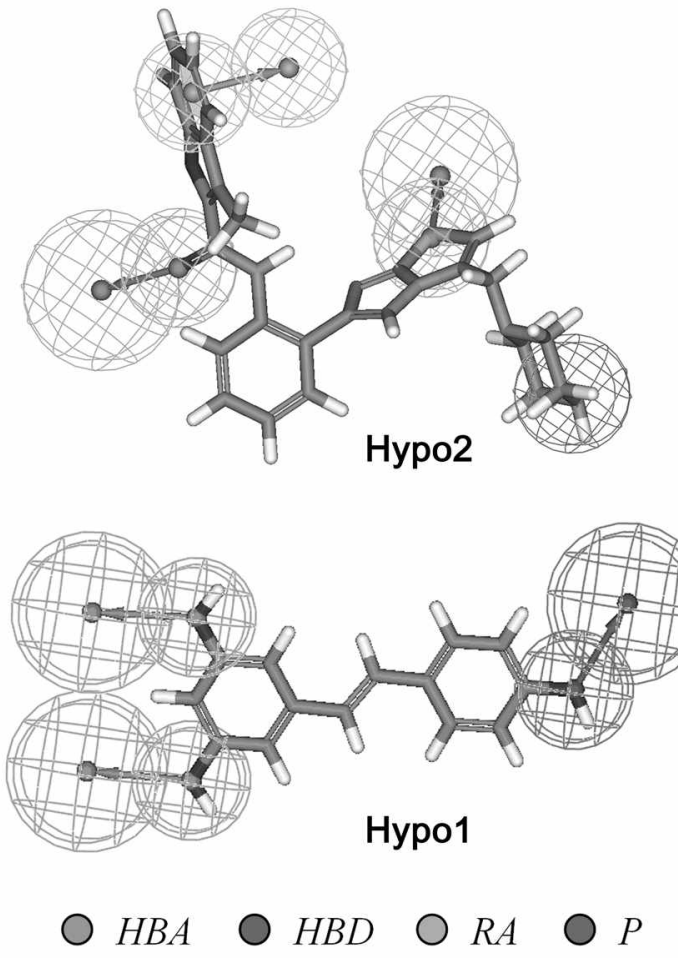

Figure 2. Comparative view of Hypo2 (4-feature) phamacophore and Hypol (3-teature).

role we further refined $\mathrm{Hyp}$ o 2

In the nest modification. $H B D$ and the $H$ groups are included and obliterated RA group in Hypo3. The hypothesis screened 5 molecules out of 176 molecules from A set and 1 molecule out of 191 molecules from $M$ set but it failed to screen any molecules from both LA and IA sets (Fig. 3). Hypo3 screened only snall number of molecules ( $3 \%$ ) from A set but it could not screen any molecules from IA set. The comparison of Hypo3 with Hypol and Hypo 2 showed that the percentage of the molecules screened from the A set was relatively very sntall. From this observation we suggest that the $H B D$ group may not play any important role in SIRTl activator.

In the third modification. instead of $P$ and $R A$ features. $Z$ and $H$ features are included in Hypot (Fig. 3). This hypothesis screened 2 molecules from $M$ set but it screened the minimum number of molecules $(0.5 \%)$ from A. LA and IA sets. As a
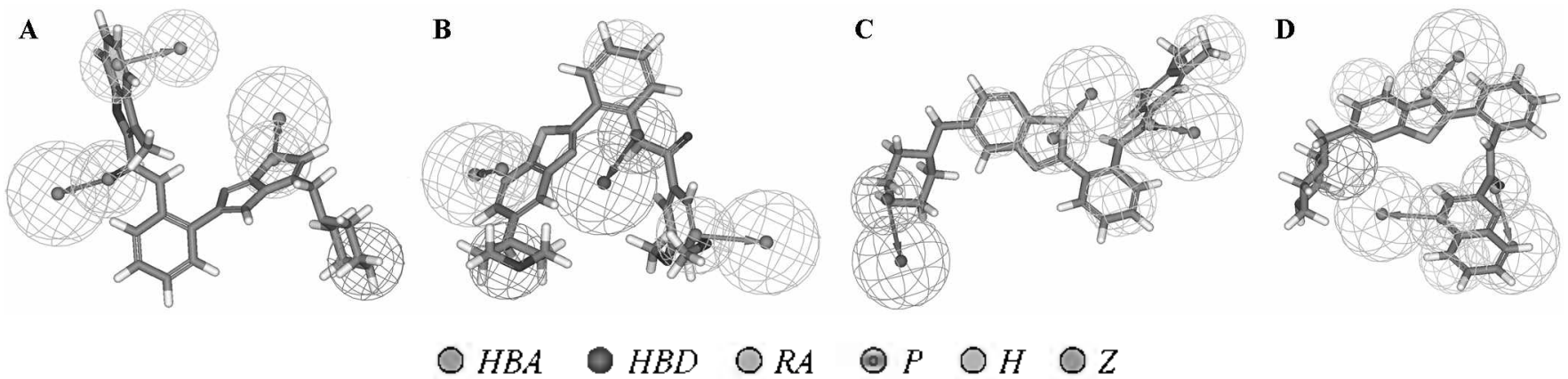

Figure 3. Comparative view of phannacophore features with molecule fit for $\mathrm{Hypo2-5}$. (A) $\mathrm{Hypo} 2$ is a four feature pharmacophore of two $H B-4$. one $R A$ and one $P$ (B) Hypo 3 is a five teature phamacophore of two $H B A$, one $H B D$, one $H$ and one $P$ (C) Hy pot is a six teature phamacophore of two $H B$. A, one $Z$, one $H B D$ and two $H$ (D) Hypos is a seven feature phamacophore of three $H B A$, one $Z$, two $H$ and one $P$. 
Table 3. Percentage comparison for the numbers of molecules screened from each hypothesis

\begin{tabular}{ccccc}
\hline \multirow{2}{*}{$\begin{array}{c}\text { Pharmaco } \\
\text { phore }\end{array}$} & \multicolumn{3}{c}{ Percentage of number of molecules screened (\%) } \\
\cline { 2 - 5 } & A set (176) & M set (191) & LA set (58) & LA set (182) \\
\hline Hypol & 11.42 & 8.90 & 10.34 & 3.86 \\
Hypo2 & 26.70 & 23.56 & 8.62 & 5.49 \\
Hypo3 & 2.84 & 0.52 & 0 & 0 \\
Hypo4 & 0.56 & 1.04 & 1.72 & 0.54 \\
Hypo5 & 0.56 & 0 & 0 & 0 \\
\hline
\end{tabular}

A Set: Active Set. M set: Moderated set. LA set: Least Active set. IA set: InActive set.

final modification. in Hypos we added $P$ and $H$ features. This hypothesis screened only one molecule from $A$ set and none from the rest of the sets (Fig. 3). By increasing the features in Hypot and 5, it screened only one molecule from the A set but the number of screened molecules were less when compared with Hypo 2 screened molecules.

Validation of Best Hypothesis. Out of these five different hypotheses. Hypol and Hypo2 screened high percentage of molecules from $\mathrm{A}$ and $\mathrm{M}$ sets, at the same time it obtained very low percentage of molecules from IA and LA sets (Table 3). The rest of the hypotheses (Hypo3-5) screened very few molecules from A set. but the percentages of the molecules screened were very low. Hypol and Hypo2 screened high numbers of molecules from $A$ and $M$ sets. However. when we compared these two hypotheses. Hypoz screened double the amount of molecules from $A$ and $M$ sets and less amount from LA and IA sets than Hypol.

$H B A$ feature was found in all the hypotheses but $H B D$ feature was only present in Hypol. In the absence of $H B D$ feature, Hypo2 screened high number of molecules from $A$ set. From this result we suggest the presence of $H B D$ feature does not affect the activity of SIRT1. By comparing the different features in all the hypotheses we have observed that $P$ and $R$ t groups in $\mathrm{Hy}$ po 2 appear to be very essential for the molecule to be an SIRT1 activator. Based on our study we propose that $\mathrm{Hypo} 2$ is the best hypothesis that consists of two $H B$.t's, one R.t and one $P$ (Fig. 3).

Virtual Screening. The best pharnacophore model, Hypo 2 was used to screen Maybridge database. The molecules that show good fit values were selected for further analysis. The best hypothesis (Hypo2) screened 57 compounds from the database and ADME (absorption, solubility and the Lipinski"s rule-of-five) properties were calculated for all the $57 \mathrm{com}-$ pounds using Discovery Studio V2.I software. In general. a molecule will be well absorbed when they posses LogP less than 5 , number of $H B A$ less than 10. number of $H B D$ less than 5. molecular weight less than 500 and number of the rotatable bond not exceeding $10{ }^{77}$ The number of rotatable bonds were considered to reduce the flexibility of the molecules. These are the important criteria to screen a good oral bioavailability dnig. Finally seven molecules have satisfied all the above criteria.

The final hit molecules were superimposed with the training set molecules for stnictural comparison (Fig. 4). Although they have different scaffolds when compared to the training

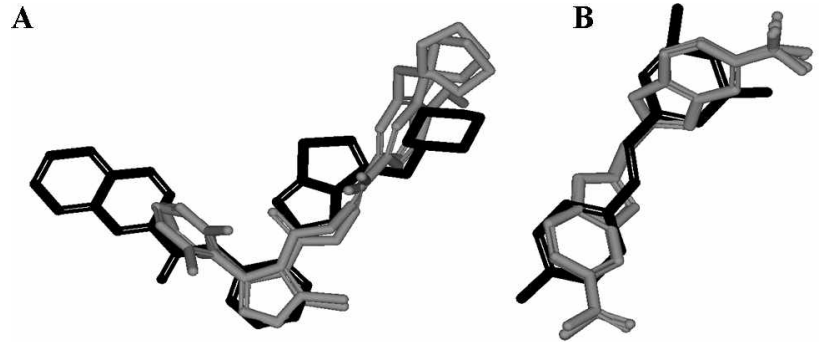

Figure 4. Structure overlap of new hit compounds with the training set molecules. SIRT 1720 with two hit compounds (SRT1720 in Black, A) and resveratrol with five hit compounds (Resveratrol in Black, B)

\section{A. $\mathrm{CD} 02704$}<smiles>COc1ccc(/C(C)=N\OC(=O)C2CSN=C2c2ccccc2Cl)cc1N1CCCCC1</smiles>

B. HTS 01501

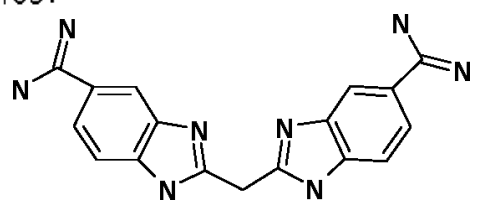

Figure 5. Two new scaftolds with show similarity with training molecules. A) First scattold shows similarity with SR'1720. B) Second scaftold shows similarity with resveratrol.

sets. those scaffolds fulfilled all the critical chenucal features presented in Hypo2. Based on the structural comparison. the final hit molecules were clustered into two groups. One group contains two molecules and this shows similarity with the SIRT1720 (Fig. 4A) and the other group contains five molecules shows similarity with resveratrol (Fig. 4B). First scaffold (CD02704) is similar to SRT1720, where the center phenyl and inidazothiazole rings were replaced by isothiazole and phenyl present in SRT1720. In the second scaffold (HTS01501). the two benzene rings in resveratrol were replaced by benzimidazole.

In SRT1720, the carbonyl and the thio group of imadazolethiazole mapped the two $H B . t$ groups and the $R A$ and $P$ mapped quinoxaline and piperazine groups. respectively. CD02704 showed the two HBA's were present in isoxazole and in linker nitrogen. $P$ was present in pyrrolidine ring and the $R A$ group was present in chlorobenzene. In HTS01501, the imidoformamide group was present in both sides of the molecule as hydroxyl group in resveratrol. The imidoformanuide groups present in HTS 01501 act as $P$ as well as $H B A$ features. The $R A$ group was present in the benzimidazole. The $H B A$ group maps the nitrogen group present in the imidazole.

Two new scaffolds were identified as SIRTl activators (Fig. 5). These two scaffolds satisfied all the critical features in Hypo 2 and they may be useful to activate SIRT1. which can be the best strategy to treat the age related diseases. 


\section{Conclusions}

A set of 3D ligand-based pharmacophore model was generated using HipHop module from CATALYST to discover the essential features of ligand, which are invaluable to examine the potential lead for SIRT 1 activation. ${ }^{2}$ Due to the lack of 3D structural information of SIRT1, protein-based pharmicophore modeling strategy could not be used for this study: Till date. there is no template for the allosteric site of SIRT1 in Protein Data Bank (www.rcsb.org). It lindered the elucidation of homology modeling and structure based pharmacophore studies for SIRT1 activators. Therefore. to identify the critical features of the SIRT l activators. ligand based pharmacophore model was used to develop the five different hypotheses. Among these five hypotheses Hypo2 (two HBA, one $R A$ and one $P$ ) screened a good number of molecules from A set. All other hypotheses screened the molecules from A set. however the number of molecules screened was too small. On the other hand. most of the molecules from IA and LA sets were failed to pass this hypothesis.

From this study. we propose that the four feature phannacophore, Hypo 2 is a good hypothesis to screen SIRT 1 activators. The best model. Hypo2 was used to screen the database in order to find out novel molecules. Totally 57 molecules were screened from the database and these molecules were further filtered using the ADME properties and Lipinski's rule of five. Seven molecules were passed all the screening strategies. Among these seven molecules. two new scaffolds were identified, which satisfied all the chemical features in Hypo2 hypothesis. These two scaffolds showed similarity in stnicture with resveratrol and SRT 1720 molecules.

Hypo 2 screened $27 \%$ of molecules from A set and $6 \%$ from IA set. Thus, we confirm that $R:$ and $P$ features are essential for the SIRT1 activators. This pharmacophore model provides key features and their relative distances. angles and geometric parameters that are important for a molecule to enliance SIRT I activity. Glucose homeostasis and insulin sensitivity in the key metabolic tissues including liver. muscle and fat were improved by SIRT1 activators. Subjecting these molecules into in vitro studies may be an excellent lead for the activation of SIRT1. SIRT 1 activation is one of the good approaches for treating age related diseases like type 2 diabetes. From this study, we concluded/suggest that the molecules having the above features will be a potent lead for the activating the SIRT1

Acknowledgments. All students were supported by a scholarship from the BK21 Program. the Ministry of Edıcation, Science and Technology. Korea and this work was supported by grants from the MOST/KOSEF for the Environmental Biotechnology National Core Research Center (grant \#:R 15-2003-012-02001-0) and M-2007-002. Daedeok Innopolis R\&BD Project.

\section{Reference}

1. Ekwall, K. Trends Genet. 2005, 11,608-615.

2. Guarente, L. Genes \& Dev. 2000, 14, 1021-1026.

3. Brain, S. C.; Tohn, D. M. Biochemisty 2006, 45, 272-282.

4. North, B. I.; Marshall, B. L.; Bora, M. T.: Denu, J. M.; Verdin. E. Mol. Cell 2003, 11, 437-444

5. Westphal, C. H.; Dipp, M. A.; Guarente, L. Thends in Bionedical Sciences 2007, 32, 555-560.

6. Olesya, V; Christian, S.; Praveen, G.; Sawa, K.; Thomas, B.; Thomas, K.: Thomas, B.: Eva, B. Circulation Research 2008 . $102,703-710$.

7. Ethan, F; Renate, V.; Gregory, L.; Comelia Magin, I. G.; Leonard, G. Genes and development 2006, 20, 1075-1080.

8. Jill, C.; Philip, D.; Simon, S.; David, P; Jesse, T; David, J.; Lei, I.; Olivier, B.: Robert, B.; Chi, B.: Pui, Y. N. Nature Lefter 2007. $450,712-716$.

9. Bjoen, S.: Eric, V. Cell Metabolism Review 2008, 7, 104-112.

10. Michael, S. F.; Till, R. D.; Nikola, P. D. Natume 2001, H10, 227-230.

11. Jill, C. M.; Philip, D. L.; Simon, S.; David, P. C.; Jesse, J. S.; David, I. G.: Lei, J.; Olivier, B.; Robert, B. P,; Chi, B. V. Iean, E. B.: Roger, X.: Jeremy, S. D.: Pui, Y. N.; Joseph, J. N.: Amy, V. L.: Hongying, Y.: Heidi, G.; Kristine, I.; Wendy, C.; Andre, I,; Siva, L.: Oliver, M.; David, A. S.; Terrold, M. O.; Michael, R. T.: Peter, J. E. Christoph, H. W. Nathe Letter. 2007, 450, 712-716.

12. Till, C. Tohn, M. Cument Opinion in Chenical Biologv 2008, 12, $11-17$

13. Porel, M: Chiarugi, A. Tiends Phamacol Sci. 2005, 26, 94-103.

14. Westphal. C. H.; Dipp, M. A.; Guarente, L. Thends in Bionedical Sciences 2001, 32, 937-944.

15. Konrad, T; Kevin, J.; Haim, Y; Dudley, W.; Jason, G.; Robert E.: David, A Kanure 2003, 425, 191-196.

16. Kaeberlein, M.: McDonagh, T.: Heltweg, B.: Hixon, J: Westman, E. A.: Caldwell, S. D.; Napper, A.; Curtis, R:; DiStefano, P. S.; Fields, S.; Bedalov, A.; Kernedv, B. K.; Denu. I. M.; J. Biol. Chent 2005, 280, 17038-17045.

17. Margie, T; Brain, C.; Tohn, M. J. Biol. Chem. 2005, 280, 17187 17195 .

18. Peter, J : Michael, J. Sirtuins Curent Opinion in Imestigational Drugs 2008, $f, 371-378$.

19. Catalyst, version 4.10; Accelrys Inc.: SanDiego, Califomia, USA, 2005

20. Jill, C.; Philip, D.: Simon, S.; David, P., Jesse, J ; David, J.: Lei, I.: Olivier, B.; Robert, B.; Chi, B.; Tean, E.: Roger, X. Teremy, S. Nature Letter 2007, 450, 712-716.

21. Brooks, B. R: Bruccoleri, R. E.; Olafson, B. D.; States, D. T.; Swaminathan, S.; Karplus, M. CHARtAA J. Comput. Chent. $1983,+187-217$

22. Yu, H.: Wang, Z; Zhang, L.: Huang, Q. Chem. Biol. Drig Des. 2007,69, 204-211

23. Vadivelan, S.; Sinha, B. N.; Irudayam, S. T.; Jagarlapudi, S. S. R. P. J. Chent. Inf. Hodel 2007, 17, 1526-1535.

24. Yu, H.: Wans, Z.: Zhang, L.; Zhang, J.: Huang, Q. Bioorg. Med. Chem. Letr. 2007, 17, 2126-2133.

25. Yuno, L.; Nagakumar, B.: Kavitha, B.; Keun, W. L. Bull. Korean Chent. Soc. 2007, 28, 561-566.

26. Wonl-Seok, O; Hang-Cheol, S.; Nak-Cheol, T.; Tae-Min, S. Bull. Korew Chent. Soc. 1996, 17, 182-188.

27. Egan, J: Merz, M: Baldwin J. J. hed. Chem. 2000, 43, $3867-3877$.

28. Akinori, H.: Masako, M: Hiroti, O.; Eiichi, K : Eiji, T.: Morifusa, E. Int. J. Hot. Sci. 2002, 3, 56-68. 